\title{
Imaging Capabilities of the Inveon SPECT System Using Single-and Multipinhole Collimators
}

\author{
Frederic Boisson ${ }^{1}$, David Zahra ${ }^{1}$, Arvind Parmar ${ }^{1}$, Marie-Claude Gregoire ${ }^{1}$, Steven R. Meikle ${ }^{2}$, Hasar Hamse ${ }^{1}$, \\ and Anthonin Reilhac ${ }^{1}$ \\ ${ }^{1}$ Australian Nuclear Science and Technology Organisation, New South Wales, Australia; and ${ }^{2}$ Brain and Mind Research Institute and \\ Faculty of Health Sciences, University of Sydney, Sydney, New South Wales, Australia
}

The Inveon small-animal SPECT system comes with several types of multipinhole collimator plates. We evaluate here the performance measurements of the Inveon SPECT system using 6 different collimators: 3 dedicated for mouse imaging and 3 for rat imaging. Methods: The measured performance parameters include the sensitivity, the spatial resolution using line sources, the ultra-micro Derenzo phantom, the recovery coefficient and the noise measurements using the National Electrical Manufacturers Association NU-4 image quality phantom, obtained with the 2 reconstruction algorithms available with the Inveon Acquisition Workplace, version 1.5-the 3-dimensional ordered-subset expectation maximization (3DOSEM) and the 3-dimensional maximum a posteriori (3DMAP). Further, the overall performance of the system is illustrated by an animal experiment. Results: The results show that the Inveon SPECT scanner offers a spatial resolution, measured at the center of the field of view, ranging from 0.6 to $1 \mathrm{~mm}$ with the collimator plates dedicated to mouse imaging and from 1.2 to less than $2 \mathrm{~mm}$ with rat collimator plates. The system sensitivity varies from 29 to $404 \mathrm{cps} / \mathrm{MBq}$ for mouse collimators and from 53 to $175 \mathrm{cps} / \mathrm{MBq}$ for rat collimators. The image quality study showed that 3DMAP allows better noise reduction while preserving the recovery coefficient, compared with other regularization strategies such as the premature termination of the 3DOSEM reconstruction or 3DOSEM followed by gaussian filtering. Conclusion: The acquisition parameters, such as the collimator set and the radius of rotation, offer a wide range of possibilities to apply to a large number of biologic studies. However, special care must be taken because this increase in sensitivity can be offset by image degradation, such as image artifacts caused by projection overlap and statistical noise due to a higher number of iterations required for convergence. 3DMAP allowed better noise reduction while maintaining relatively constant recovery coefficients, as compared with other reconstruction strategies.

Key Words: single photon emission computed tomography; smallanimal; image quality; NEMA; multi-pinhole

J Nucl Med 2013; 54:1833-1840

DOI: 10.2967/jnumed.112.117572

\footnotetext{
Received Nov. 29, 2012; revision accepted May 7, 2013.

For correspondence or reprints contact: Frederic Boisson, ANSTO LifeSciences, Imaging Group, Australian Nuclear Science and Technology Organisation, Locked Bag 2001 Kirrawee DC, New South Wales, 2232, Australia.

E-mail: E: fredericb@ansto.gov.au

Published online Sep. 5, 2013.

COPYRIGHT (C) 2013 by the Society of Nuclear Medicine and Molecular Imaging, Inc.
}

$\mathbf{S}$ -animal SPECT systems typically use pinhole collimation, which presents a conical geometry, leading to high magnification and high spatial resolution (1-3). Higher detection efficiency can be obtained using multipinhole collimation (4-9) without theoretically trading the resolution. The Inveon small-animal SPECT system (Siemens Medical Solution) comes with several types of multipinhole collimator plates, which can be mounted on the detector heads. These collimators are designed to rotate at specific radii of rotation (RORs). The combination of the collimator geometric design (number and placement of the pinholes, collimator length, aperture, and degree of multiplexing) and the ROR used fixes many characteristics such as the active field of view (FOV) and the performance of the system in terms of resolution, sensitivity, and resulting reconstruction image quality (IQ). This is different from PET imaging, for which there are no collimation-related parameters to optimize for each specific task that is determined by the end-user. It is therefore fundamentally important to characterize the performance of the system obtained with each available collimation profile before conducting any biologic studies. Previous work has already been reported for this system with 2 single pinholes designed for mouse imaging (10). In the present paper, we evaluated the performance measurements of the Inveon SPECT system using 6 different collimators: 3 dedicated for mouse imaging (1 single-pinhole and 2 with 5 pinholes) and 3 for rat imaging ( 1 single-pinhole and 2 with 3 pinholes). The measured performance parameters include the sensitivity; the spatial resolution; and the IQ, namely the recovery coefficient (RC) and noise measurements, obtained with the 2 available reconstruction algorithms - the 3-dimensional ordered-subset expectation maximization (3DOSEM) and the 3-dimensional maximum a posteriori (3DMAP). Further, the overall performance of the system is illustrated by an animal experiment.

\section{MATERIALS AND METHODS}

\section{System Description}

The imaging system evaluated in this study is the SPECT component of the Inveon multimodality small-animal imaging system. The Inveon multimodality scanner combines SPECT, PET, and CT components in a common gantry, in which the SPECT and CT modules, mounted perpendicular to each other on a rotating platform, are placed at the front and the PET module at the rear. The SPECT system comprises $2 \gamma$-detector heads facing each other, each composed of a $68 \times 68$ pixelated scintillator array of $2 \times 2 \times 10 \mathrm{~mm}$ thallium-doped sodium iodide crystals with a $0.2-\mathrm{mm}$ crystal gap. The detector heads are coupled to a $3 \times 3$ array of position-sensitive photomultiplier 
tubes, leading to a maximum active region of $150 \times 150 \mathrm{~mm}$. The energy range of the system is $20-300 \mathrm{keV}$, which disables the possibility of performing imaging of high-energy $\gamma$ radiomolecules and simultaneous imaging of single photon- and positron-emitting radiomolecules as described in the study by Goorden et al. (11). An energy resolution of $12.4 \%$ was previously reported for this system by Magota et al. (10) using ${ }^{99 \mathrm{~m}} \mathrm{Tc}$. We measured an energy resolution of $13.9 \%$ using ${ }^{57} \mathrm{Co}$. Three types of collimators are available and can be mounted on each detector: the mouse pyramid, which comes with 6 different mouse pinhole collimator plate sets; the rat pyramid, which comes with 3 different rat pinhole collimator plates; and finally the low-energy, all-purpose parallel-hole collimator. Table 1 presents parameters of all the single- and multipinholes available with the Inveon SPECT component. The collimator plate is maintained on the pyramid with powerful magnets, and the pyramid is itself attached to the detector via 2 draw latches and 2 pins to ensure that the collimator can be accurately reattached. A dedicated geometric calibration file is used for every collimator set. The collimators are designed to work at 1 of 3 RORs, the distance from the center of rotation to the center of the pinhole.

In this study, we focused only on assessing the performance obtained with the 6 collimator sets marked in Table 1: the single pinhole with a $0.5-\mathrm{mm}$ aperture (1-MHR-0.5) and the 2 multipinhole sets dedicated for mouse imaging (5-MBR-0.5 and 5-MWB-1) (where MHR is mouse high resolution, MBR is mouse brain, and MWB is mouse whole body) and the single pinhole with a 1.5-mm (1-RGP-1.5) aperture and the 2 multipinhole sets dedicated for rat imaging with 1.2- (3-RWB-1.2) and 1.8-mm (3-RWB-1.8) apertures. The studied collimators present transaxial FOVs varying from 90 to $110 \mathrm{~mm}$ for the rat and from 26 to $60 \mathrm{~mm}$ for the mouse (ROR-dependent) and axial FOVs varying from 90 to $130 \mathrm{~mm}$ for the rat and from 30 to 90 $\mathrm{mm}$ for the mouse. The axial FOV can also be extended up to $250 \mathrm{~mm}$ using continuous bed motion, an acquisition mode that is not studied here. SPECT data are acquired in list-mode format to support postacquisition rebinning for gated studies and energy discrimination. Finally, 2 different methods are available to reconstruct the images, 3DOSEM and 3DMAP.

\section{Performance Studies}

Unless specified otherwise, projections were acquired in the stepand-shoot mode with 60 views over $360^{\circ}$ at $30 \mathrm{~s}$ per projection. The acquisition time per view was automatically corrected according to the radioactive decay of ${ }^{99 \mathrm{~m}} \mathrm{Tc}$, leading to a total acquisition time of 32 min. Rebinning was done using a $20 \%$ energy window centered at the $140-\mathrm{keV}$ photopeak of ${ }^{99 \mathrm{~m}} \mathrm{Tc}$. A correction matrix derived from a point source flood scan was used to normalize the reconstructed images, and no attenuation or scatter correction was performed. The Inveon Acquisition Workplace (IAW) 1.5 was used for all SPECT reconstructions (3DOSEM/3DMAP) using resolution modeling.

Tomographic Spatial Resolution. Tomographic spatial resolution was measured for all the collimator plates using a line source (inner diameter, $0.28 \mathrm{~mm}$ ) filled with ${ }^{99 \mathrm{~m}} \mathrm{Tc}$ solution and placed in the center of the transaxial FOV. Resolution was measured for all the RORcollimator combinations. The 3DOSEM algorithm was used to reconstruct the images, with 8 iterations and 5 subsets and a voxel size set to $0.2 \times 0.2 \times 0.2 \mathrm{~mm}$. The count profiles through the voxel with the highest counts from the reconstructed transaxial images were plotted in both horizontal and vertical directions. The full width at half maximum (FWHM) and full width at tenth maximum were calculated using gaussian fits of the resulting count profiles. The results were not corrected for the spatial extent of the sources.

An ultra-micro Derenzo phantom was also scanned to visually evaluate the reconstructed spatial resolution obtained with the tested collimators. With an inner diameter of $28 \mathrm{~mm}$, the phantom consists of fillable hot rods with different diameters $(0.75,1.00,1.35,1.70,2.00$, and $2.40 \mathrm{~mm}$ ) arranged into 6 segments. The phantom was filled with $115 \mathrm{MBq}$ of ${ }^{99 \mathrm{~m}} \mathrm{Tc}$ solution, leading to a concentration of $16.42 \mathrm{MBq} /$ $\mathrm{mL}$. For all the tested collimators, projection data were acquired at an ROR of $35 \mathrm{~mm}$ for the mouse collimators and $50 \mathrm{~mm}$ for the rat collimators. The 3DOSEM algorithm was used to reconstruct the images, with 8 iterations and 5 subsets and a voxel size set to $0.2 \times$ $0.2 \times 0.2 \mathrm{~mm}$.

System Sensitivity. System sensitivity was measured in planar mode for each collimator plate using a ${ }^{99 \mathrm{~m}} \mathrm{Tc}$ point source and for different RORs. The point source consisted of a microsieve on which $2 \mathrm{MBq}$ of ${ }^{99 \mathrm{~m}} \mathrm{Tc}$ solution had been deposited. The point source was then positioned in the center of the axial and transaxial FOVs. Each time, a 20min acquisition was performed to ensure that a total of at least 10,000 counts were collected for each measurement. Following the National Electrical Manufacturers Association (NEMA) procedure (12), the system sensitivity was defined as the ratio of the recorded counts per second and the decay-corrected activity of the point source.

\section{IQ}

The NU-4 IQ phantom (12) was used to study the convergence performance of the 2 proposed reconstruction methods and ultimately to characterize the quality of the resulting reconstructed images. The phantom was filled with a ${ }^{99 m} \mathrm{Tc}$ solution with an initial activity of 111

TABLE 1

Single and Multipinholes Available with Inveon SPECT System

\begin{tabular}{|c|c|c|c|c|}
\hline Collimation type & Diameter of aperture $(\mathrm{mm})$ & Name & $\mathrm{ROR}(\mathrm{mm})$ & Focal length (mm) \\
\hline \multicolumn{5}{|l|}{ Mouse } \\
\hline \multirow[t]{4}{*}{ Single pinholes } & 0.5 & 1-MHR-0.5* & 25,30 , and 35 & 97.2 \\
\hline & 1 & 1-MGP-1 & 25,30 , and 35 & NM \\
\hline & 2 & 1-MHS-2 & 25,30 , and 35 & NM \\
\hline & 3 & 1-MME-3 & 30,35 , and 40 & NM \\
\hline \multirow[t]{2}{*}{ Multipinholes } & $5 \times 0.5$ & $5-M B R-0.5^{\star}$ & 25,30 , and 35 & 94.3 \\
\hline & $5 \times 1$ & $5-M W B-1^{*}$ & 30,35 , and 40 & 89.9 \\
\hline \multicolumn{5}{|l|}{ Rat } \\
\hline Single pinhole & 1.5 & 1-RGP-1.5* & 50,55 , and 60 & 70 \\
\hline \multirow[t]{2}{*}{ Multipinholes } & $3 \times 1.2$ & $3-R W B-1.2^{*}$ & 50,55 , and 60 & 70.4 \\
\hline & $3 \times 1.8$ & 3-RWB-1.8* & 50,55 , and 60 & 70.5 \\
\hline
\end{tabular}

${ }^{*}$ Collimator sets used in present study.

$\mathrm{NM}=$ not measured. 
$\mathrm{MBq}(5 \mathrm{MBq} / \mathrm{mL})$ for the acquisitions using the mouse collimators and with an initial activity of $74 \mathrm{MBq}(3.33 \mathrm{MBq} / \mathrm{mL})$ for the acquisitions using the rat collimators. For all the tested collimators, projection data were acquired at an ROR of $35 \mathrm{~mm}$ for the mouse collimators and $50 \mathrm{~mm}$ for the rat collimators. Data were reconstructed with 3DOSEM and 3DMAP into image matrices with a voxel size set to $0.5 \times 0.5 \times 0.5 \mathrm{~mm}$. Multiple reconstructions were performed with different parameters to evaluate their effect on the IQ. For 3DOSEM, we varied the number of iterations between 1 and the number of iterations for which convergence (percentage difference below $0.5 \%$ between 2 consecutive iterations) was observed for the mean activity measured in the rods. The impact of postreconstruction filtering on the IQ was also studied. For 3DMAP, we used the number of iterations for which convergence was observed, and we varied the strength of the prior ( $\beta$-parameter) from 1 to 10 (the maximum allowed value) with increments of 1 . Two metrics were used to quantify the quality resulting from the different reconstruction approaches and associated parameters: the RCs and the image noise, both estimated from the resulting reconstructed images. For that purpose, a cylindric volume of interest (VOI) matching the physical volume of the uniform region of the IQ phantom was drawn. The average concentration in this VOI and the SD were measured to estimate the noise. Ten cylindric VOIs were also drawn around each rod, with a diameter equal to the physical diameter and covering the central 10-mm length of the rods. The values in each of these VOIs were measured and divided by the mean value obtained in the uniform region to find the RCs for each rod size.

\section{Animal Study}

Finally, $2 \mathrm{BALB} / \mathrm{c}$ mice were injected with a solution of $99 \mathrm{~m} \mathrm{Tc}-$ sestamibi and scanned immediately after injection for $3 \mathrm{~h}$, one using the 3-RWB-1.2 collimators (injected dose, 25.2 MBq) at an ROR of 50 $\mathrm{mm}$ and the second using the 5-MWB-1 collimators (injected dose, $34.7 \mathrm{MBq}$ ) at an ROR of $30 \mathrm{~mm}$. Because of the shorter axial FOV, however, the 5-MWB-1 set required a helical scan to enable a wholebody acquisition. For both experiments, a 30-min acquisition, $30 \mathrm{~min}$ after injection, was performed in step-and-shoot mode. The data obtained with the 5-MWB-1 collimator were reconstructed into image matrices of $88 \times 88 \times 132$ voxels of $0.5 \mathrm{~mm}$ in all directions, resulting in image sizes of $44 \times 44 \times 66 \mathrm{~mm}$. The data obtained with the 3 RWB-1.2 collimator were reconstructed into image matrices of $180 \times$ $180 \times 220$ voxels of $0.5 \mathrm{~mm}$ in all directions, resulting in image sizes of $90 \times 90 \times 110 \mathrm{~mm}$.

The mice were euthanized at the end of the scanning time. The same reconstruction settings as in the phantom experiment were applied to enable a direct comparison of the phantom IQ results with those obtained for the mouse images. No attenuation or scatter corrections were performed.

${ }^{99 \mathrm{~m} T c-s e s t a m i b i}$ is a well-known cardiac imaging radiotracer used in clinical practice. Besides this, it is also used to study expression of P-glycoprotein (13). This protein is expressed in all major organs, such as the liver, kidneys, bowels, and capillaries of the blood-brain barrier, and tumors as well.

Animal experiments were performed in compliance with the National Health and Medical Research Council Australian Code of Practice for the care and use of animals for scientific purposes and sanctioned by the Australian Nuclear Science and Technology Organisation Animal Care and Ethics Committee.

\section{RESULTS}

\section{Physical Studies}

Figure 1 shows the tomographic spatial resolution (FWHM) as a function of the sensitivity measured for all the tested collimator sets. Values are plotted for all the corresponding RORs. The measured spatial resolution ranged from $0.57 \mathrm{~mm}$ (1-MHR-0.5) to $1.98 \mathrm{~mm}$ (3-RWB-1.8), and the system sensitivity was found to range between $29.3 \mathrm{cps} / \mathrm{MBq}(1-\mathrm{MHR}-0.5)$ and $403.6 \mathrm{cps} / \mathrm{MBq}$ (5-MWB-1). Sensitivities and resolution values for other commercial micro-SPECT scanners-the U-SPECT-II $(14,15)$ and the nanoSPECT $(14,16)$ - are also reported in Figure 1, and the

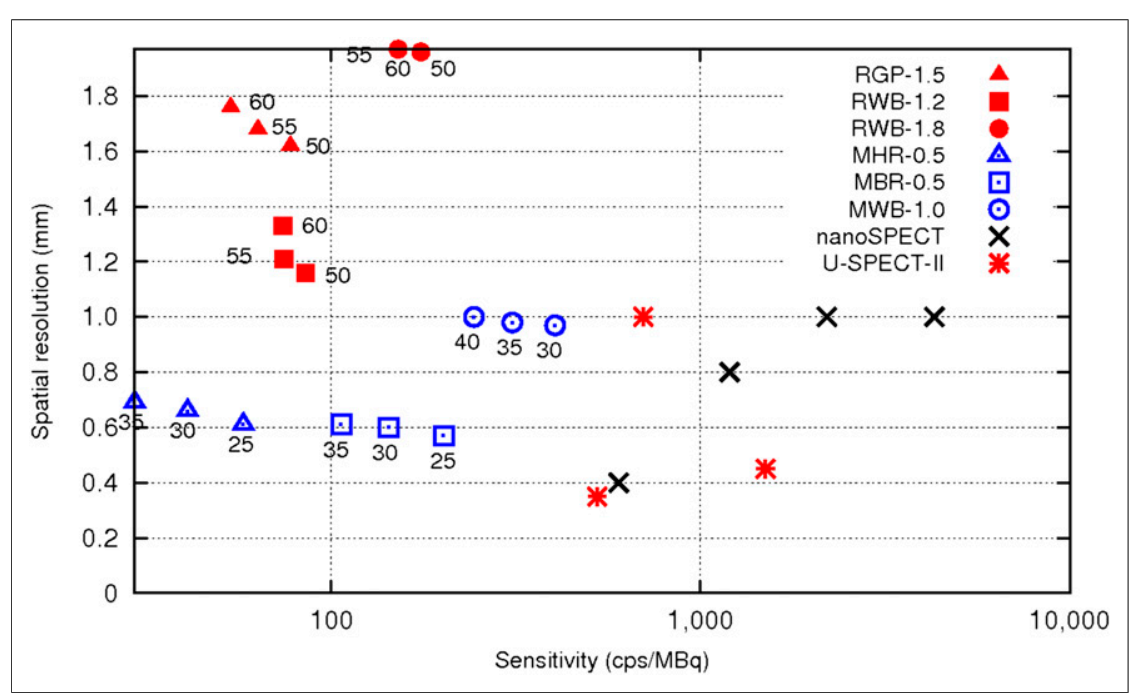

FIGURE 1. Tomographic spatial resolution as function of sensitivity measured with tested rat and mouse collimator sets: 1-RGP-1.5, 3-RWB-1.2, 3-RWB-1.8, 1-MHR-0.5, 5-MBR0.5 , and 5-MWB-1.0. RORs at which sensitivity and resolution measurements were performed are indicated in figure. Performance for 2 other micro-SPECT scanners, U-SPECT-II and nanoSPECT, are also reported for comparison. For U-SPECT-II, ultrahigh-resolution whole-body/focused mouse collimator (UHR-M), with aperture of $0.35 \mathrm{~mm}$; general-purpose collimator for mouse (GP-M), with aperture of $0.6 \mathrm{~mm}$; and ultrahigh sensitivity collimator for mouse (UHS-M), with aperture of $1 \mathrm{~mm}$, were used. For nanoSPECT, UHR-M collimator, with aperture of $0.6 \mathrm{~mm}$, and HR-M, with aperture of $1 \mathrm{~mm}$, were used. comparison is detailed in the "Discussion" section. The resolution values reported for the U-SPECT-II and the nanoSPECT systems (15) with the ultrahigh-resolution collimators were obtained using a pixel size below $0.13 \mathrm{~mm}$ in place of the $0.2 \mathrm{~mm}$ in our study.

The measured values of spatial resolution using the different collimators were also studied using the 3DOSEM reconstructed images of the micro-Derenzo phantom shown in Figure 2 (3DMAP Derenzo images obtained with the mouse collimators are shown in Supplemental Fig. 1; supplemental materials are available at http://jnm.snmjournals.org). The 1-mm hot rods can be visually distinguished with the 1-MHR-0.5 and with the 5-MBR-0.5 mouse plates, whereas this segment is hardly resolved with the 5-MWB-1 mouse plates. The 1.7-mm hot rods can be distinguished with the 3 rat collimator plates.

\section{IQ}

Figure 3 shows the absolute percentage differences of the activity measured between 2 consecutive 3DOSEM/3DMAP $(\beta=10)$ 


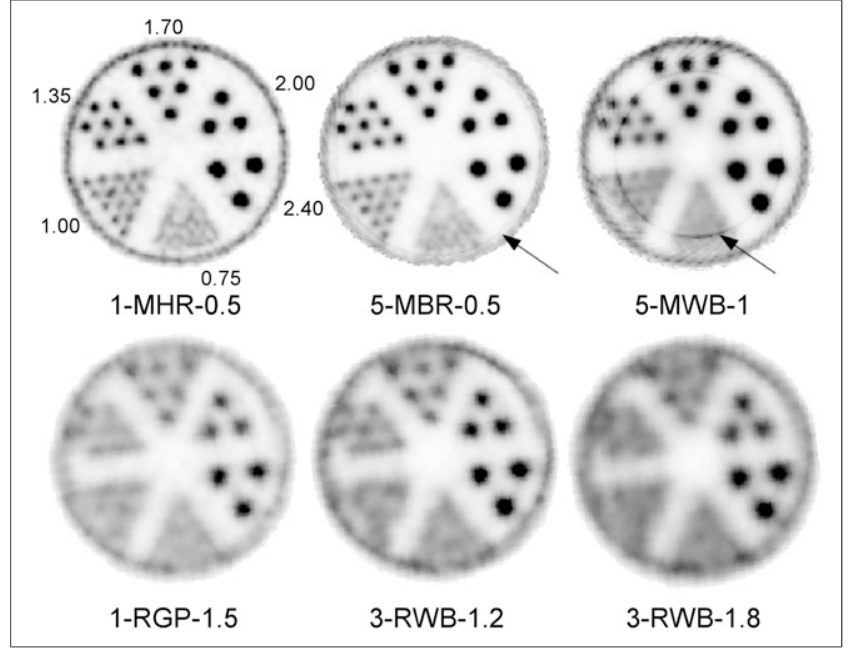

FIGURE 2. Derenzo phantom images obtained with 6 studied collimators. Observe ring artifacts obtained with 2 mouse 5-pinhole collimator plates due to limited FOV.

iterations in the 2- and the 5-mm rods of the IQ phantom obtained with the tested mouse and rat collimator sets. This convergence study showed that percentage differences of the activity measured in each insert between 2 consecutive iterations fell below $0.5 \%$ when 20,35 , and 100 iterations ( 5 subsets) were used for the reconstruction of MHR, MBR, and MWB scans, respectively, for both 3DOSEM and 3DMAP reconstruction methods and for the full range of prior strength $([1,10])$. The convergence for the 1-mm rod could not be established using the 2 mouse multipinhole collimator plates because the measured recovery values in this rod were close to 0 and subject to background fluctuations (see the "Discussion" section and Supplemental Fig. 3). The convergence results obtained with the rat collimator plate sets showed that percentage differences of the activity measured in each insert between 2 consecutive iterations fell below $0.5 \%$ when 50 3DOSEM iterations (5 subsets) were used. However, only 8 iterations were required to reach convergence for rods with a diameter greater than $1 \mathrm{~mm}$. The convergence properties of 3DOSEM (and 3DMAP) differ depending on the mouse collimator plates used (Figs. 3A and 3B). This is not the case for projections acquired with the rat collimators (Figs. 3C and $3 \mathrm{D}$ ), for which the convergence properties are similar when no prior is used. However, still for the rat collimators, the use of a prior slightly accelerated the convergence for the small rods.

$\mathrm{RCs}$ measured at convergence for the mouse and the rat collimator sets are shown in Figure 4 (RCs measured at convergence with 3DMAP and different prior strengths are presented in Supplemental Fig. 2). The RCs measured for the smallest 1-mm rod were 0.02 and 0.045 for the 5-MBR-0.5 mouse collimator and for the 3-RWB-1.8 rat collimator, respectively, whereas for the 5$\mathrm{mm}$ rod the RCs were 0.74 and 0.78 for the 1-MHR- 0.5 mouse collimator and the 3-RWB-1.2 rat collimator, respectively. Multipinhole rat collimators demonstrated higher RCs than multipinhole mouse collimators, whereas single-pinhole rat collimators yielded smaller RCs than single-pinhole mouse collimators.

Figure 5 shows the RCs for the 2- and 5$\mathrm{mm}$ rods as a function of noise (STD computed from the homogeneous region) for different reconstruction strategies and for the tested collimator plate sets: 3DOSEM with increasing number of iterations (until convergence), 3DMAP until convergence with increasing prior strength (1-10), and 3DOSEM until convergence with a postreconstruction gaussian filtering step $(\sigma$ ranging from 0.5 to $4.5 \mathrm{~mm}$ with a $1-\mathrm{mm}$ increment). 3DMAP reconstruction gives better image properties than 3DOSEM either using premature termination (few iterations) or using 3DOSEM until convergence followed by gaussian filtering. This is particularly true for projections acquired with the mouse collimator plates for which the use of a prior efficiently reduces the noise without significantly affecting the resolution (as reported with the RCs). The results even suggest that the use of a prior increases the RCs for the small rods. In addition, the convergence obtained with 3DOSEM in the rods using the mouse multipinhole collimators is not monotonic, and a minimum is reached after few iterations. The convergence is object-dependant and in our case not homogeneous because of the activity distribution in the phantom. It is recommended that for mouse imaging, one should use a higher number of iterations or

3DMAP.
FIGURE 3. Absolute percentage differences of activity measured between 2 consecutive 3DOSEM/3DMAP $(\beta=10)$ iterations in 2- and 5-mm rods of IQ phantom obtained with tested mouse and rat collimator sets. 


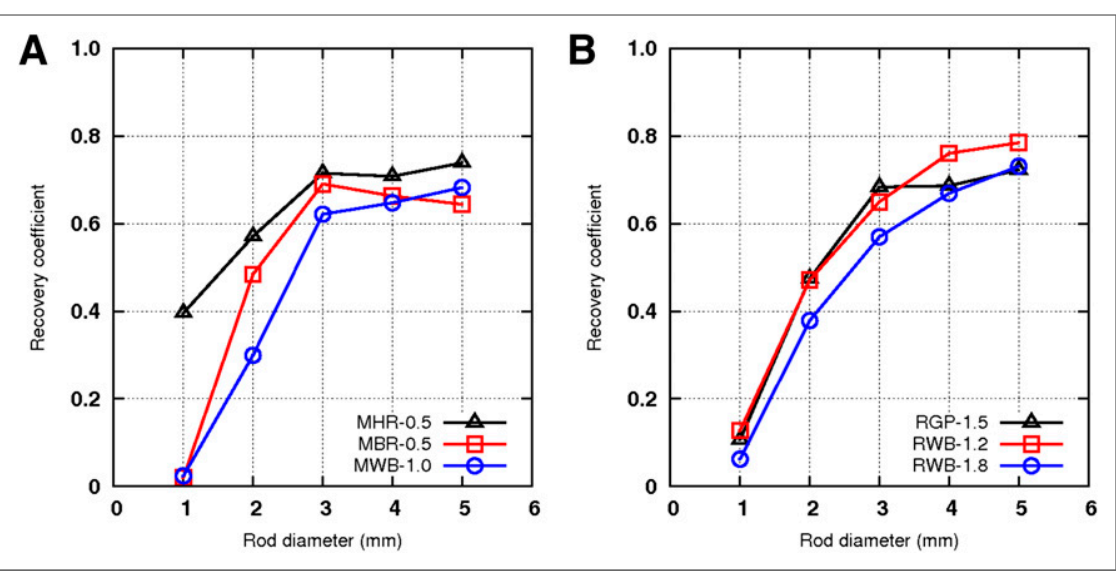

FIGURE 4. RCs measured at convergence from 3DOSEM images and for mouse $(A)$ and rat $(B)$ collimator sets.

High degrees of projection overlap and truncation are observable with the 2 mouse multipinholes, whereas for this emitting object size, a low overlap of the projections is observable with the multipinhole rat collimators. Truncations in the axial dimension are still observable using the multipinhole rat collimators. When not iterated sufficiently, 3DOSEM provided suboptimal IQ, exhibiting band artifacts and ring artifacts in transaxial images. After 4 iterations, the activity level in the homogeneous regions is recovered (still with the artifacts), whereas the activity in the rod is not (Supplemental Fig. 4). This nonhomogeneous convergence explains the nonmonotonic convergence shown in Figure $5 \mathrm{~A}$, where a higher number of iterations reduces the magnitude of the artifacts at a cost of increased noise. 3DMAP provides images with attenuated artifacts and reduced noise.

\section{Animal Study}

Coronal sections of 2 mice injected with a solution of ${ }^{99 \mathrm{~m}} \mathrm{Tc}$-sestamibi and scanned immediately after injection for $3 \mathrm{~h}$ using the 5-MWB-1 collimator set at an ROR of $30 \mathrm{~mm}$ and the 3RWB-1.2 collimator at an ROR of $50 \mathrm{~mm}$ are shown in Figures $6 \mathrm{~A}$ and $6 \mathrm{~B}$, respectively.

Figure 6A was obtained with 3DMAP using 100 iterations (5 subsets) and a prior of 10 . Figure $6 \mathrm{~B}$ was obtained with 3DOSEM
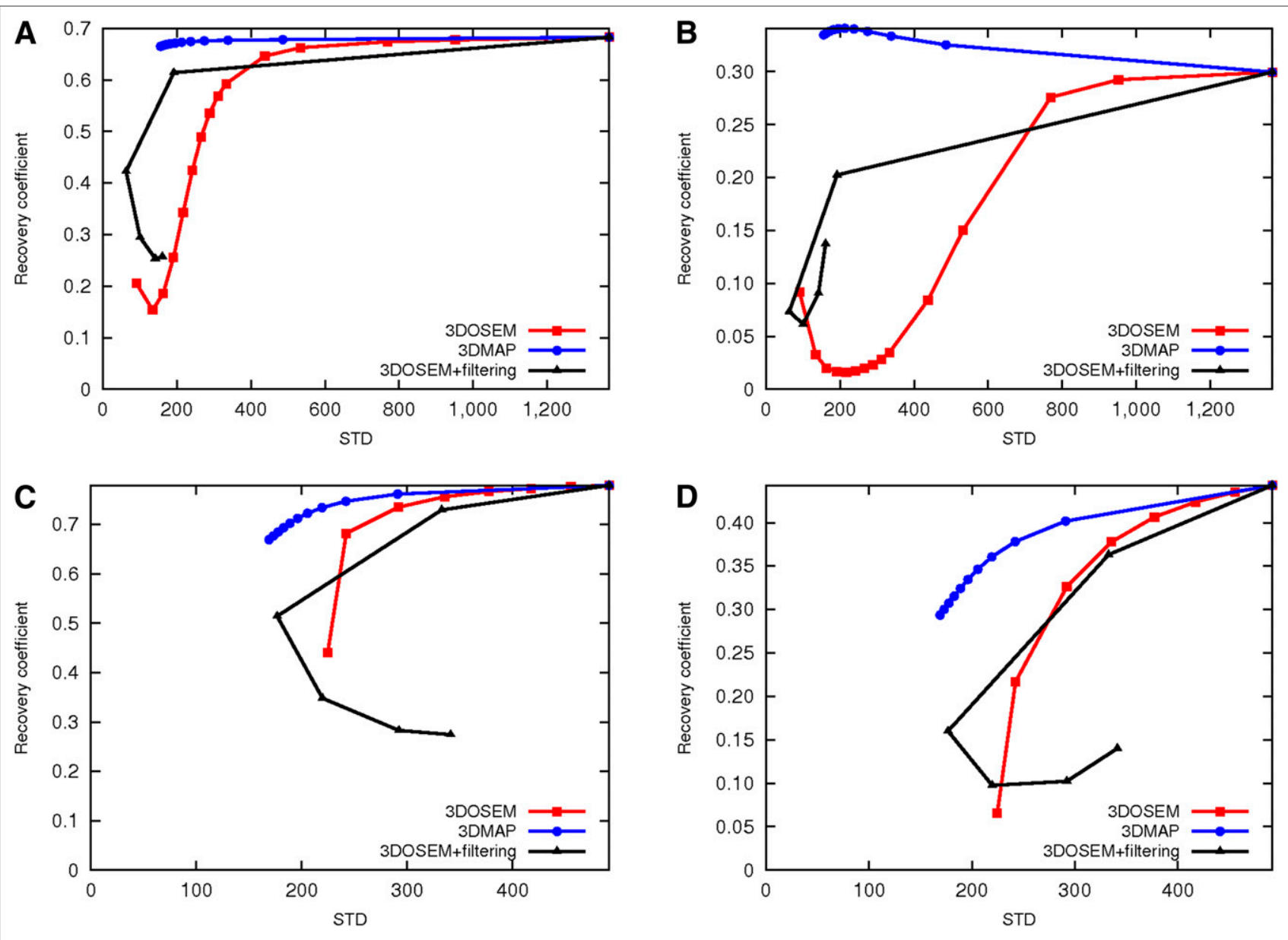

FIGURE 5. (A and B) RCs obtained with 5-MWB-1 mouse collimator for 5- and 2-mm rods vs. SD measured from 3DOSEM, 3DMAP, and 3DOSEM followed by gaussian smoothing images, respectively. ( $C$ and $D)$ RCs obtained with 3-RWB-1.2 rat collimators for 5- and 2-mm rods vs. SD measured from 3DOSEM, 3DMAP, and 3DOSEM followed by gaussian smoothing images, respectively. 


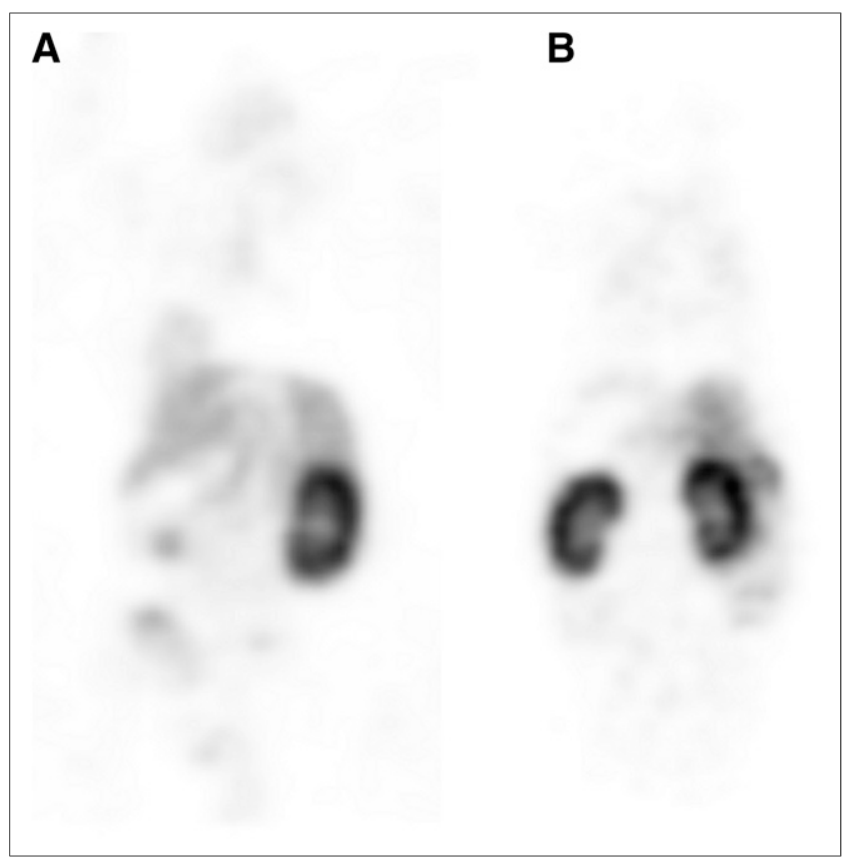

FIGURE 6. $\quad{ }^{99 m}$ Tc-sestamibi images in mice obtained with collimator plates dedicated for mouse $(A)$ and rat $(B)$ imaging.

using 8 iterations (5 subsets) and no postreconstruction filtering. Coronal slices were chosen to show kidney uptake on both animals to provide a qualitative comparison.

\section{DISCUSSION}

We characterized the Inveon SPECT system for different acquisition and reconstruction settings and for different collimator sets, among which 3 were dedicated to mouse imaging and 3 to rat imaging. The measured performance included the spatial resolution, sensitivity, noise, and RCs.

The resolution study showed that the aperture of the pinhole fixes the spatial resolution (17). With the 2 mouse multipinhole collimator sets (5-MBR-0.5 and 5-MWB-1) and the 3-RWB-1.8, the ROR does not seem to affect the spatial resolution as measured in the center of the field of view, whereas the lower the ROR the better the resolution with the 1-MHR-0.5, 1-RGP-1.5, and 3-RWB1.2 plates. Spatial resolution values of $0.84,1.03$, and $1.08 \mathrm{~mm}$ in FWHM at RORs of 25,30 , and $35 \mathrm{~mm}$ were previously reported in Magota et al. (10) for the 1-MHR-0.5 collimator plates, in place of $0.61,0.66$, and $0.69 \mathrm{~mm}$ in our study. We attribute the differences in reported values to the use of different versions of the acquisition and reconstruction software (IAW, version 1.2.2.2, in Magota et al. (10); IAW, version 1.5, in the present study). Indeed, we confirmed this by reconstructing the data using the same version as was used in Magota et al. (10), which resulted in resolution values comparable to those reported in that study.

The resolution measured with a line source at the center of the scanner and using an iterative reconstruction method does not accurately reflect the resolution obtained when imaging a real object. Indeed, the spatial resolution can degrade quickly when we move outward from the center. Also, the image properties obtained with iterative reconstruction, such as resolution, depend on the distribution of the activity within the FOV. The visual assessment of the resolution using the ultra-micro Deluxe Derenzo phantom is likely to provide resolution estimates closer to the actual values obtained during animal imaging studies.

We found that spatial resolution assessed with the Derenzo phantom was systematically inferior to that obtained with the line source measurements. The Derenzo phantom image using the 1-MHR-0.5 and reconstructed with IAW, version 1.2.2.2, with a 0.5 -mm pixel size could not resolve properly the $1.35-\mathrm{mm}$ quadrant as reported in Magota et al. (10), whereas in our study, the 1-mm rods could be distinguished when using IAW, version 1.5, and a $0.2-\mathrm{mm}$ pixel size. The reconstruction of the Derenzo images using a $0.5-\mathrm{mm}$ pixel size with IAW, version 1.5 , led to the same conclusion (images not shown), showing again that better IQ is obtained with the newer version of the software. These results suggest that the improvement is due to a more accurate resolution modeling in the system matrix used during the reconstruction step. Using hot rod phantoms, Deleye et al. (15) showed that capillaries as small as $0.4,0.45$, and $0.6 \mathrm{~mm}$ could be resolved with the U-SPECT-II with 75 pinholes of $0.35 \mathrm{~mm}$, the U-SPECT-II with 75 pinholes of $0.6 \mathrm{~mm}$, and with the nanoSPECT with 36 pinholes of $0.6 \mathrm{~mm}$, respectively.

As expected, sensitivity increases were obtained using multipinhole collimation in place of single-pinhole collimator plates. For the same ROR and aperture, the result shows that the sensitivity obtained with the 5 pinholes is 3.5 times that obtained with a single pinhole with the same aperture size. The increase is therefore not strictly equal to the number of holes because the acceptance angles are different between the 1-MHR-0.5 and the 5-MBR-05 but most importantly because the use of the 5-MBR-05 induces projection truncations as observed in Figure 6. Still, using the 1MHR-0.5 and the 5-MBR-0.5 results, this sensitivity increase is obtained without trading away the spatial resolution as shown in Figure 1 and on the Derenzo images in Figure 2. The results from the IQ study show that these increases in sensitivity obtained with overlapping projections do not necessarily translate into objective improvements in IQ as discussed below.

Comparison of the sensitivities obtained with the Inveon and other commercial systems shows that the Inveon scanner systematically offers lower sensitivity. This lower sensitivity has to be mitigated by the fact that the tested SPECT component from the trimodal Inveon system (which contains 3 modules: PET, SPECT, and CT combined in a single gantry) has only 2 detector heads in place of 4 for the dedicated Inveon SPECT system. This makes a difference with the nanoSPECT, which contains 4 detector heads, or the USPECT-II, which is a stationary full-ring system. Sensitivity obtained with the 4-detector Inveon SPECT system would be twice the reported values without altering the spatial resolution.

The remaining differences in sensitivity between the nanoSPECT and the Inveon SPECT can be explained by the design of the collimator plates. Although collimator plates with up to 16 pinholes are available with the nanoSPECT (and with unknown degree of multiplexing), only 3 and 5 pinholes are currently available with the Inveon SPECT system for rat and mouse imaging, respectively. A higher number of pinholes as in the nanoSPECT may increase the sensitivity with a higher degree of multiplexing, which may not be translated to images with better quality. The comparison of the nanoSPECT and the Inveon SPECT with the U-SPECT-II system is not fair to the latter system because the 2 first systems use overlapping projections, resulting in an artificial increase of the system sensitivity but with a significant amount of information per photon lost because of the overlap. However, the 
U-SPECT-II uses nonoverlapping projections, resulting in true system sensitivity measurements (14). It is also important to note that micro-SPECT systems also vary widely in the FOV. Therefore, a fair comparison of system performance is not straightforward. The sizes of the FOV, associated with the possible contamination of the activity present outside the latter, can lead to artifacts in the reconstructed image. In this study, we found that the use of rat collimators in the case of mouse studies may be a wise choice.

Unlike PET systems dedicated to small-animal imaging, for which NEMA has defined the standard NU 42008 (12), SPECT systems are, to date, subject to no standards. A task force has recently been assigned to establish what could be the new NEMA NU 5, also including a new phantom, adapted to small-animal SPECT systems and to define the IQ parameters. In anticipation of this possible NEMA NU 5, the IQ phantom (NU 4 IQ phantom) defined by the NU 42008 standard was used in previous studies to characterize the performance of small-animal SPECT scanners and, more particularly, the IQ $(10,18)$.

One goal of this study was to examine the convergence properties of the reconstruction algorithms with the tested collimators. We first observed that more iterations were required to reach convergence (and maximum RCs) for the smaller rods because high frequencies converge more slowly than low frequencies. Harteveld et al. (18) reported the same observation for the U-SPECT-II using also the IQ phantom.

As expected, the number of iterations sufficient to reach convergence with the IQ phantom scans was found to be different depending on the collimator sets (Fig. 3). We observed that data obtained with rat multipinhole collimators yield faster convergence than data obtained with mouse multipinhole collimators. This is likely resulting from differences in the degree of multiplexing obtained with the different collimator plates. Because of a lower degree of multiplexing for the studied emitting object with rat collimators, the convergence properties were found to be less dependent on the plates used (Fig. 3). Multiplexing poses ambiguous information for reconstructions because counts in overlapping regions cannot be traced back through a specific pinhole. This uncertainty causes an enlargement of the ensemble of possible solution images increasing the required number of iterations for convergence and the statistical noise in the solution image. This can be seen in Figure 5 where 100 iterations were required to reach convergence with the 5-MWB-1 collimator, compared with 8 for the 3-RWB-1.2. The resulting image noise, estimated from the homogeneous region, is about 3 times higher (coefficient of variation more than 5 times higher), despite the higher initial activity $(111 \mathrm{MBq}$ in place of $74 \mathrm{MBq}$ ) and higher sensitivity (more than 3 times). In addition, when too few iterations are performed, iterative reconstruction of overlapping and truncated projections provides images with suboptimal quality, exhibiting band and ring artifacts as seen with the multipinhole collimators for mouse imaging. Artifacts can be reduced using a higher number of iterations at a cost of higher noise. 3DMAP reconstruction seems to provide a viable alternative. Although much smaller, ring artifacts could be seen also with the rat multipinhole collimators. This is in agreement with recent studies (19-22) from which it is clear that improved detection efficiency by increased multiplexing is offset by image degradation due to increased noise and apparent band and ring artifacts.

Convergence could not be established for the 1-mm rod when using the mouse multipinholes because the activity values were extremely low and subject to background fluctuations. Conse- quently, RCs obtained for the 1-mm rod with the multipinhole mouse plates as reported in Figure 3 were close to null whereas the spatial resolution study showed that (at least) the 5-MBR-0.5 collimator should allow us to resolve the 1-mm rod. Projection overlap and truncation in the case of multipinhole apertures explain this result. The Derenzo images shown in Figure 2 do not show this effect. However, the Derenzo phantom was centered in the FOV, whereas the IQ phantom was axially offset (with regard to the rod portion) to have both the rods and the homogeneous region in the FOV. Ring artifacts were visible on the Derenzo images when acquired with the mouse multipinhole collimators (Fig. 2).

More generally, projection truncation and overlap and restricted FOV with multipinholes explain why the RCs obtained with the 5MBR-0.5 are different from those obtained with the single pinhole (1-MHR-0.5), whereas according to the resolution study, the RC should be similar. Although the IQ phantom is larger than a typical mouse but much smaller than a rat, it still provides quantitative and objective data on the effect of different reconstruction and acquisition settings. However, the number of iterations obtained in the present study may not be truly predictive for the number of iterations needed for a mouse or rat scan, which need some specific adjustments. All this is in line with the proposal of Harteveld et al. (18) about the development of a NEMA NU 5 standard for SPECT and, more specifically, the use of an additional phantom with smaller internal structures and smaller outer diameter to better mimic mouse acquisitions such as the one proposed by Visser et al. (23).

RCs obtained with the single pinhole (1-MHR-0.5) differ again from the values reported in Magota et al. (10) obtained with the same experimental settings except for the reconstruction platform version. The version used in this present study (version 1.5) allows a much better recovery of the small rods $(\mathrm{RC}=0.4$ for the $1-\mathrm{mm}$ rod, in place of $<0.2$ reported in Magota et al. (10) using version 1.2.2.2), while giving slightly lower RCs for a higher rod diameter (rod diameter, $>3 \mathrm{~mm}$ ). The RCs shown in Figure 5 demonstrate that structures bigger than $2 \mathrm{~mm}$ can be similarly resolved using rat collimator plates in place of mouse collimator plates, with the former providing a bigger FOV. This is illustrated with the wholebody images of a mouse obtained with 5-MWB-1 at an ROR of $30 \mathrm{~mm}$ and 3-RWB-1.2 at an ROR of $50 \mathrm{~mm}$ (Figs. 6A and 6B, respectively).

The IQ study showed that 3DMAP-compared with other regularization strategies such as the premature termination of the 3DOSEM reconstruction or 3DOSEM followed by gaussian filtering-allowed better noise reduction while preserving the RC. This is particularly true for the mouse collimator plates for which RCs were preserved while the noise is efficiently reduced using a strong prior weight. Although this is less obvious with rat collimator plates, 3DMAP reconstruction still resulted in better performance than the other reconstruction strategies. These results indicate that 3DMAP using optimal parameters should be preferred over 3DOSEM. We observed that the spatial filtering with a high kernel size can result in an increase of the noise and of the RCs (Fig. 5). This is likely due to the 3D nature of the spatial filtering, which causes an activity gradient in the axial direction, mainly due to spillover from the homogeneous region onto the rod region.

\section{CONCLUSION}

This study evaluated the performance of the small-animal SPECT Inveon system using different collimator sets dedicated to mouse 
and rat imaging. The acquisition parameters, such as the collimator set and the ROR, offer a wide range of possibilities to apply to a large number of biologic studies. The results show that the Inveon SPECT system offers spatial resolution, measured at the center of the FOV, ranging from 0.6 to $1 \mathrm{~mm}$ with the collimator plates dedicated to mouse imaging and from 1.2 to less than $2 \mathrm{~mm}$ with rat collimator plates. The system sensitivity varies from 29 to $404 \mathrm{cps} / \mathrm{MBq}$ for mouse collimators and from 53 to $175 \mathrm{cps} / \mathrm{MBq}$ for rat collimators. The use of multipinholes increased the system sensitivity. However, special care must be taken because this increase in sensitivity can be offset by image degradation, such as image artifacts caused by projection overlap and statistical noise due to a higher number of iterations required for convergence. 3DMAP allowed better noise reduction while maintaining relatively constant RCs as compared with other reconstruction strategies.

These IQ parameters can guide the researcher in the choice of optimal imaging settings in real small-animal experiments, including the choice of the collimator that will provide the most acceptable results. From this current set of experiments, we concluded that, despite a lower sensitivity and spatial resolution, rat multipinhole collimator plates are more appropriate for wholebody imaging of mice than the mouse whole-body multipinhole plates, because the reconstructed images have a larger FOV and present reduced artifacts, less statistical noise, and similar signal recovery.

\section{DISCLOSURE}

The costs of publication of this article were defrayed in part by the payment of page charges. Therefore, and solely to indicate this fact, this article is hereby marked "advertisement" in accordance with 18 USC section 1734 . No potential conflict of interest relevant to this article was reported.

\section{REFERENCES}

1. Schramm NU, Ebel G, Engeland U, et al. High-resolution SPECT using multipinhole collimation Trans Nucl Sci. 2003;50:315-320.

2. Franc BL, Acton PD, Mari C, et al. Small-animal SPECT and SPECT/CT: important tools for preclinical investigation. J Nucl Med. 2008;49:1651-1663.

3. Weber DA, Ivanovic M. Ultra-high-resolution imaging of small animals: implications for preclinical and research studies. J Nucl Cardiol. 1999;6:332-344.

4. Vunckx K, Beque D, Defrise M, et al. Single and multipinhole collimator design evaluation method for small animal SPECT. IEEE Trans Med Imaging. 2008;27: $36-46$.
5. Cao Z, Bal G, Accorsi R, et al. Optimal number of pinhole in multi-pinhole SPECT for mouse brain imaging-a simulation study. Phys Med Biol. 2005;50:4609-4624.

6. Beekman FJ, van der Have F, Vastenhouw B, et al. U-SPECT-I: a novel system for submillimeter-resolution tomography with radiolabeled molecules in mice. J Nucl Med. 2005;46:1194-1200.

7. Vanhove C, Defrise M, Lahoutte T, et al. Three-pinhole collimator to improve axial spatial resolution and sensitivity in pinhole SPECT. Eur J Nucl Med Mol Imaging. 2008;35:407-415.

8. Difilippo FP. Design and performance of a multi-pinhole collimation device for small animal imaging with clinical SPECT and SPECT-CT scanners. Phys Med Biol. 2008;53:4185-4201.

9. Meikle SR, Kench PL, Weisenberger AG, et al. A prototype coded aperture detector for small animal SPECT. IEEE Trans Nucl Sci. 2002;49:2167-2171.

10. Magota K, Kubo N, Kuge Y, et al. Performance characterization of the Inveon preclinical small-animal PET/SPECT/CT system for multimodality imaging. Eur J Nucl Med Mol Imaging. 2011;38:742-752.

11. Goorden MC, van der Have F, Kreuger R, et al. VECTor: a preclinical imaging system for simultaneous submillimeter SPECT and PET. J Nucl Med. 2013;54: 306-312.

12. National Electrical Manufacturers Association (NEMA). Performance Measurements of Small Animal Positron Emission Tomographs. NEMA standards publication NU4-2008. Rosslyn, VA: National Electrical Manufacturers Association; 2008.

13. Piwnica-Worms D, Chiu M, Budding M, et al. Functional imaging of multidrugresistant Pgp with an organotechnetium complex. Cancer Res. 1993;53:977-984.

14. van der Have F, Vastenhouw B, Ramakers RM, et al. U-SPECT-II: an ultra-highresolution device for molecular small-animal imaging. J Nucl Med. 2009;50: 599-605.

15. Deleye S, VanHolen R, Verhaeghe J, et al. Performance evaluation of smallanimal multipinhole $\mu$ SPECT scanners for mouse imaging. Eur J Nucl Med Mol Imaging. 2013;40:744-758.

16. Schramm N, Hoppin J, Lackas C, et al. Improving resolution, sensitivity and applications for the NanoSPECT/CT: a high-performance SPECT/CT imager for small-animal research [abstract]. J Nucl Med. 2007;48(suppl 2):436P.

17. Anger HO. Scintillation camera. Rev Sci Instrum. 1958;29:27-33.

18. Harteveld AA, Meeuwis APW, Disselhorst JA, et al. Using the NEMA NU 4 PET image quality phantom in multipinhole small-animal SPECT. J Nucl Med. 2011; 52:1646-1653.

19. Mok GSP, Tsui BMW, Beekman FJ. The effects of object activity distribution on multiplexing multi-pinhole SPECT. Phys Med Biol. 2011;56:2635-2650.

20. Vunckx K, Suetens P, Nuyts J. Effect of overlapping projections on reconstruction image quality in multipinhole SPECT. IEEE Trans Med Imaging. 2008;27: 972-983.

21. Mahmood ST, Erlandsson K, Cullum I, et al. The potential for mixed multiplexed and non-multiplexed data to improve the reconstruction quality of a multi-slitslat collimator SPECT system. Phys Med Biol. 2010;55:2247-2268.

22. Kench PL, Lin J, Gregoire MC, et al. An investigation of inconsistent projections and artefacts in multi-pinhole SPECT with axially aligned pinholes. Phys Med Biol. 2011;56:7487-7503.

23. Visser EP, Harteveld AA, Meeuwis APW, et al. Image quality phantom and parameters for high spatial resolution small-animal SPECT. Nucl Instrum Meth A. 2011;654:539-545. 